\title{
Motivación e intención de ser físicamente activo en jugadores de baloncesto en formación. Diferencias en función de la competición
}

\author{
Motivation and intention to be physically active in basketball \\ players in training. Differences depending on the competition
}

\author{
Evelia Franco, Javier Pérez-Tejero y Aintzane Arrizabalaga
}

PALABRAS CLAVE: Baloncesto, iniciación, formación, motivación, liga RESUMEN: El objetivo del presente estudio fue analizar las posibles diferencias en la satisfacción de las necesidades psicológicas básicas (NPB) y la intención de la práctica futura en jugadores de baloncesto en formación según la liga en la que competían. Se administró, a una muestra de 181 jugadores de baloncesto entre 11 y 16 años pertenecientes a ligas diferentes (juegos deportivo municipales, federado y federado preferente), el cuestionario PNSE para evaluar las necesidades psicológicas básicas y el MIFA para medir la intención de ser físicamente activo. Los resultados más significativos indicaron que a) existen diferencias significativas entre ligas; b) la relación entre las NPB y la intención aumentaba con el nivel de la liga; c) la competencia era la única variable presente en todas las categorías estudiadas.
KEY WORDS: Basketball, initiation, training, motivation, league ABSTRACT: The objective of this study was to analyse the differences in the satisfaction of basic psychological needs (BPNS) and intended future practice in basketball players in training according to the level of competition to which they belonged. It was distributed PNSE questionnaire to assess basic needs and MIPA to measure the intention to be physically active on a sample of 181 basketball players aged between 11 and 16 years belonging to different leagues. The most significant results indicated that a) there are significant differences between leagues b) the relation between the BPN and the intention increased with the level of competition c) competence was the only variable present in all categories.

\section{Introducción}

La motivación del deportista hacia la práctica en la que esta involucrado es un factor que determina su futura continuidad en el desarrollo de actividad físico - deportiva (Sarrazin, Vallerand, Guillet, Pelletier, y Cury, 2002). La teoría de la Autodeterminación (Deci y Ryan, 1985; Ryan y Deci, 2000) aporta una base conceptual para explicar esta relación, estableciendo que la motivación intrínseca, directamente relacionada con conductas que buscan la diversión, el interés y complacencia inherente a la actividad, actúa como un predictor para la permanencia (Vallerand 1997; Ntoumanis 2005; Moreno, Cervelló, González-Cutre, 2007; Navarro, González-Cutre et al. 2008), mientras que la desmotivación es causa del abandono (Sarrazin, Vallerand, Guillet, Pelletier y Cury, 2002). La persistencia en la actividad física puede ser generada por un alto nivel de motivación autodeterminada; por el contrario, la frustración puede provocar la desmotivación del deportista y llevar al abandono. Esta teoría también establece que el grado de satisfacción de las necesidades psicológicas básicas (NPB) (autonomía, competencia y relación) actúa como mediador del nivel de autodeterminación de los practicantes (Kilpatrick, Hebert y Jacobsen 2002; González-

Dirección para correspondencia: Evelia Franco Álvarez. Facultad de Ciencias de la Actividad Física y del Deporte. Universidad Politécnica de Madrid. C/ Martín Fierro, 7. 28040 MADRID.

E-mail: e.francoalvarez@gmail.com
Cutre, Moreno et al. 2005; García Calvo 2006) y, en consecuencia, puede predecir la persistencia o abandono de la práctica deportiva.

Pocos estudios han abordado este tema en el ámbito deportivo. En los existentes, la intención de persistir en la práctica deportiva viene asociada a altos niveles de competencia (García Calvo, Sánchez Miguel, Leo, Sánchez Oliva y Amado, 2012), de autonomía (Almagro, Sáenz-López y Moreno, 2010; García Calvo, Sánchez Miguel et al. 2012) y de relación (García Calvo et al 2011).

El objetivo del presente estudio es analizar las posibles diferencias en la satisfacción de las necesidades psicológicas básicas y en la intención de la práctica futura en jugadores de baloncesto en formación según a la liga en la que participan.

\section{Método}

La muestra estaba formada por 181 jugadores de baloncesto en formación, con edades comprendidas entre 11 y 16 años $(\mathrm{M}=14.25$; DT=1.23). Del total de la muestra, el $25.4 \%$ jugaba en juegos deportivos municipales, el $44.8 \%$ en liga federada y el $29.8 \%$ en federada preferente. Los jugadores tenían una experiencia media de 5.52 años $(\mathrm{DT}=2.62)$ en la práctica del baloncesto.

El material utilizado para la realización del presente estudio fue la versión adaptada al español (Moreno, Marzo, 
y Martínez Galindo, 2011) de la "Escala de medición de la satisfacción de las necesidades psicológicas en el ejercicio (PNSE; Wilson, Rogers, Rodgers, y Wild, 2006)" creada para evaluar la satisfacción de las necesidades psicológicas básicas (autonomía, competencia y las relaciones con los demás) y la "Medida de la Intencionalidad para ser Físicamente Activo (MIFA; Heins y cols, 2004)", adaptada al castellano (Moreno, Moreno, y Cervelló (2007).

En un primer lugar se realizó un análisis descriptivo de los datos. Posteriormente, y con el objeto de apreciar posibles diferencias entre las categorías objeto de estudio, se utilizó un análisis de la varianza. Para estudiar posibles diferencias entre categorías concretas, se utilizó el ajuste para comparaciones múltiples de Scheffé En todos los casos se utilizó un nivel de significación de $\mathrm{p}<.05$.

\section{Resultados}

La tabla 1 muestra los estadísticos descriptivos obtenidos en las variables analizadas de acuerdo a la competición en la que participan los jugadores. Se observa la existencia de diferencias en la satisfacción de la necesidad de relación $(\mathrm{p}<.05)$ y en la intencionalidad de práctica físico-deportiva $(\mathrm{p}<.05)$ futura según la competición en la que participen los jugadores.

La tabla 2 muestra las correlaciones bivariadas encontradas entre las variables de acuerdo a la competición en la que participan los jugadores. La intención de ser físicamente activo se relacionó positivamente con la competencia en todas las competiciones y con la relación en federada preferente. Por otra parte, en federado la competencia se relacionó con el resto de variables.

Tabla 1. - Estadísticos descriptivos de la satisfacción de las necesidades psicológicas básicas y de la intencionalidad de práctica futura según la liga a la que pertenezcan los jugadores

\begin{tabular}{lccccccc}
\hline \multirow{2}{*}{ VARIABLES } & \multicolumn{2}{c}{$\begin{array}{c}\text { JUEOS DEPORTIVOS } \\
\text { MUNICIPALES }\end{array}$} & \multicolumn{2}{c}{ FEDERADOS } & \multicolumn{2}{c}{$\begin{array}{c}\text { FEDERADOS PREFE- } \\
\text { RENTE }\end{array}$} \\
\cline { 2 - 7 } & Media & D. T. & Media & D. T. & Media & D .T. & \\
\hline COMPETENCIA & 3.58 & 0.80 & 3.82 & 0.73 & 3.98 & 0.53 & .323 \\
AUTONOMÍA & 2.75 & 0.70 & 3.03 & 1.17 & 2.90 & 0.84 & .301 \\
RELACIÓN & 3.66 & 0.44 & 3.64 & 0.62 & 3.96 & 0.57 & $.034^{*}$ \\
INTENCIÓN & 4.19 & 0.69 & 4.33 & 0.67 & 4.52 & 0.55 & $.042^{*}$ \\
\hline
\end{tabular}

* Significativo al nivel .05

Los análisis a posteriori revelan que los jugadores pertenecientes a equipos que compiten en categoría federada preferente $(M=3.96$; $D T=0.57)$ muestran mayores puntuaciones en la variable relación que los jugadores que pertenecen a equipos que lo hacen en otras categorías federadas $(M=3.64$;
DT=0.62) o en los juegos deportivos municipales $(M=3.66$; $\mathrm{DT}=0.44)$; y también presentan niveles más elevados de intención de práctica físico-deportiva futura $(\mathrm{M}=4.52$; $\mathrm{DT}=0.55)$ que aquellos pertenecientes a juegos deportivos municipales $(\mathrm{M}=4.19 ; \mathrm{DT}=0.69)$.

Tabla 2. Correlaciones bivariadas entre intención de práctica y las necesidades psicológicas básicas de acuerdo a la competición en la que participan

\begin{tabular}{ccccccccccccc}
\hline & \multicolumn{4}{c}{ Juegos deportivos municipales } & \multicolumn{3}{c}{ Federados } & \multicolumn{3}{c}{ Federados preferente } \\
\hline & $\mathrm{C}$ & $\mathrm{A}$ & $\mathrm{R}$ & $\mathrm{I}$ & $\mathrm{C}$ & $\mathrm{A}$ & $\mathrm{R}$ & $\mathrm{I}$ & $\mathrm{C}$ & $\mathrm{A}$ & $\mathrm{R}$ & $\mathrm{I}$ \\
$\mathrm{C}$ & - & .136 & .091 & $.556^{* *}$ & - & $.262^{*}$ & $.407^{* *}$ & $.224^{*}$ & - & -.079 & .257 & $.322^{*}$ \\
$\mathrm{~A}$ & .136 & - & .051 & .069 & $.262^{*}$ & - & -.080 & .182 & -.079 & - & -.133 & .043 \\
$\mathrm{R}$ & .091 & .051 & - & .147 & $.407^{* *}$ & -.080 & - & .167 & .257 & -.133 & - & $.364^{*}$ \\
$\mathrm{I}$ & $.556^{* *}$ & .069 & .147 & - & $.224^{*}$ & .182 & .167 & - & $.322^{*}$ & .043 & $.364^{*}$ & - \\
\hline
\end{tabular}

$\mathrm{C}=$ Competencia; $\mathrm{A}=$ Autonomía; $\mathrm{R}=$ Relación; $\mathrm{I=Intención}$

\section{Discusión}

La competencia es, de las tres necesidades básicas, la que aparece relacionada, o como predictor, de la intención de ser físicamente activo en todos los estudios consultados; y así a sucedido en las tres categorías estudiadas, siendo explicado por el hecho de que un jugador que se sienta competente en el contexto deportivo se sentirá motivado a seguir practicando. 
La segunda variable que, junto con la competencia, suele aparecer asociada a la intención es la relación. En nuestro estudio este hecho solo se presenta en liga preferente, coincidiendo con los resultados obtenidos por García-Calvo et al. (2011) y García Calvo et al. (2012) en una población practicante de deportes colectivos y rango de edad igual al de nuestra muestra; y por Sarrazin et al. (2002) con una muestra de jugadoras de balonmano de edades similares. El que solo aparezca en la liga de mayor nivel competitivo podría deberse a que la dificultad para pertenecer a estos equipos genere una mayor sentimiento de pertenencia al grupo de iguales y permita plantearse la posibilidad de poder pasar en un futuro al baloncesto de elite.

De lo observado en el estudio se puede sugerir, como futuro tema de análisis, el planteamiento de estrategias, en los clubs que tienen varias categorías, que generen entre los deportistas de las inferiores, mejores expectativas sobre el futuro en la práctica.

\section{Referencias}

Almagro, J., Sáenz-López, P., González Cutre Coll, D., y Moreno Murcia, J. A. (2010). Clima motivacional percibido, necesidades psicológicas y motivación intrínseca como predictores del compromiso deportivo en adolescentes. Revista Internacional de Ciencias del Deporte., 7, 250 - 265.

Deci, E. L., y Ryan, R. M. (1985). Intrinsic motivation and self-determination in human behaviour. New York: Plenum.

García Calvo, T. (2006). Motivación y comportamientos adaptativos en jóvenes futbolistas., Universidad de Extremadura.

García Calvo, T. (2011). Incidencia de la teoría de autodeterminación sobre la persistencia deportiva. Revista Internacional de Ciencias del Deporte., $\operatorname{VII}(25), 226-276$.

García Calvo, T., Sánchez Miguel, P. A., Leo, F. M., Sánchez Oliva, D., y Amado, D. (2012). Análisis del grado de diversión e intención de persistencia en jóvenes deportistas desde la perspectiva de la teoría de la autodeterminación. Revista de Psicología del Deporte, 21(1), 7-13.

González-Cutre, D., Moreno, J. A., Conte, L., Martínez Galindo, C., Alonso Villodre, N., Zomeńo, T., et al. (2005). Análisis de la motivación autodeterminada en jóvenes deportistas a través del clima motivacional percibido en los iguales y las orientaciones de meta.

Hein, V., Müür, M. y Koka, A. . (2004). Intention to be physically active after school graduation and its relationship to three types of intrinsic motivation. European Physical Education Review, 10(1), 5-19.

Kilpatrick, M., Hebert, E., y Jacobsen, D. (2002). Physical activity motivation: A practitioner's guide to self-determination theory. Journal of Physical Education, Recreation \& Dance, 73(4), 36-41.

Moreno, J. A., Cervelló, E., y González-Cutre, D. (2007). Young athletes' motivational profiles. Journal of Sports Science and Medicine, 6, 172-179.
Moreno, J. A., Marzo, J. C., \& Martínez Galindo, C. (2011). Validación de la escala de "Satisfacción de las necesidades psicológicas básicas" y del cuestionario de la "Regulación conductual en el deporte" al contexto español. International Journal of Applied Sports Sciences, 7(26), 355-369.

Moreno, J. A., Moreno, R., \& Cervelló, E. (2007). El autoconcepto físico como predictor de la intención de ser físicamente activo. Psicología y Salud, 17(2), $261-267$.

Navarro, N., González-Cutre, D., Marcos, P. J., Borges, F., Hernández, A., Vera, J. A., et al. (2008). Perfiles motivacionales en la actividad física saludable: un estudio desde la perspectiva de la teoría de la autodeterminación. In U. P. d. Olavide (Ed.), Actas del XI Congreso Nacional, XI Andaluz y III Iberoamericano de Psicología de la Actividad Física y del Deporte. Sevilla.

Ntoumanis, N. (2005). A Prospective Study of Participation in Optional School Physical Education Using a Self-Determination Theory Framework. [Article]. Journal of Educational Psychology, 97(3), 444-453.

Ryan, R. M., y Deci, E. L. (2000). Self-determination theory and the facilitation of intrinsic motivation, social development, and well-being. American Psychologist, 55(1), 68-78.

Sarrazin, P., Vallerand, R., Guillet, E., Pelletier, L., y Cury, F. (2002). Motivation and dropout in female handballers: A 21-month prospective study. European Journal of Social Psychology, 32, 395-418.

Vallerand, R. (1997). Toward a hierarchical model of intrinsic and extrinsic motivation. Advances in Experimental Social Psychology 29, 271 - 360.

Wilson, P., Rogers, W., Rodgers, W., y

Wild, T. (2006). The psychological need satisfaction in exercise scale. Journal of Sport and Exercise Phychology, 28, 231-251. 